\title{
EDITORIAL
}

\section{Oxidative Stress and the Biological and Chemical Processes in Health}

\section{THE ROLE OF OXIDATIVE STRESS}

Oxidative stress is the result of reactive oxygen species (ROS) overproduction or/and a decline in antioxidant defense mechanisms. ROS are generated by highly reactive elements with unpaired electrons e.g. $\mathrm{O}_{2}-$ (superoxide radical), $\mathrm{OH}$ (hydroxyl radical) and $\mathrm{H}_{2} \mathrm{O}_{2}$ (hydrogen peroxide). Overall excessive generation of ROS results in deleterious effects causing damage to DNA, proteins, and lipids, ultimately leading to cell death (JARRET et al., 2008; SANTOS et al., 2011). Therefore, agents counteracting excess ROS and/or increasing the antioxidant defenses represent an appealing strategy for the treatment of different complications (DOS SANTOS et al., 2019). On the other hand, ROS have important functions in cellular signaling as participants and modifiers of signaling pathways, essential for the proper development and proliferation of cells. Indeed, ROS play an important role in the immunologic system and on the increment of the antioxidant response (DOS SANTOS et al., 2019). For instance, physical exercise, a therapeutic approach for several diseases, induce the production of ROS, leading to an increase in the antioxidant defense in rest condition (Jl et al., 1988).

The current understanding of the complex role of ROS is necessary for developing multi-functional antioxidants, which can maintain ROS in a non-pathological level. In this context, numerous research groups have focused on the characterization of the ROS source and their pathway triggered. Therefore, multi-aspects ROS in health and disease have been addressed in the current issue. This special issue includes 8 original research and review articles focusing on the role of ROS and antioxidant defense on the development of diabetes and its associated diseases. The guest editors are pleased to present a selective group of these cutting-edge original research and review articles as follows.

In the review "Mitochondrial biochemistry and oxidative stress in bipolar disorder: New horizons" the author discusses recent articles on the effect of ROS on Bipolar Disorder (BD), a disease characterized by high suicide rates. Mitochondria is a specialized organelle that generates adenosine triphosphate but also is considered the primary source of ROS. Mitochondrial ROS plays a critical role in BD. Recent literature consistently reported changes in antioxidant enzymes, lipid peroxidation, and nitric oxide levels induced by in BD.

In the original article "ginger hydroethanolic extract inhibits oxidation of low-density lipoprotein (LDL) in-vitro" the authors used an isolated fraction of LDL of patient serum samples, the LDL fraction was isolated, previously treated with Ginger Rhizome Hydroethanolic Extract (EHEG). They concluded that ginger has protective activity against the formation of oxidized LDL, suggesting that its phytochemical compounds confer antioxidant activity, and may be effective in the prevention and/or treatment of cardiovascular diseases, especially those related to the process of atherosclerosis.

In the original article "The effect of fish oil supplementation on sepse" the authors induced sepsis by puncture and cecal ligation after several days of fish oil (FO) administration in rats. Follow they assessed lipid peroxidation, lung and renal histology were analyzed regarding its structure and presence of polymorphonuclear (PMN), lung edema index, renal function and survival rate. They concluded that FO supplementation, in the dose and period studied, did not improve renal, pulmonary and mortality rate in rats with sepsis.

In the article "Antioxidant effect of LLes paraguariensis and condiments of lipid oxidation of oreochromis niloticus steak" aimed to evaluate the antioxidant potential of yerba mate extract, in association with rosemary, oregano and thyme condiments, in tilapia fillets submitted to different preparation forms, in face of lipid oxidation of 
fish. The extracts were evaluated for the percentage reduction of the 2,2-diphenyl-1-picryl-hydrazyl radical and for the content of total phenolic compounds. The lipid oxidation of the fish was analyzed by thiobarbituric acid reactive substances (TBARS) test. Based on the result, they suggest the effectiveness of the association of condiments with yerba mate in the reduction of oxidative rancidity of the tilapia fillets submitted to the different thermal processes applied, as well as the in natura product.

In the review of literature "The consumption of fruits as a potential alternative to accelerate the recovery process after high intensity exercise" the authors discussed the recent literature that used fruits to abbreviate the recovery process after high intensity exercise in different sports modalities by reducing ROS and muscle damage markers. According to the authors, nutrient supplementation can enhance recovery after high intensity exercise, particularly fruits of red-blue color such as cherries and blueberries seem to decrease oxidative stress, inflammation, and muscle damage.

In the original article "In-vitro effect of Camellia sinensis extract in human DNA leukocytes" the authors aimed to evaluate the possible genotoxic activity of the green tea extract through the evaluation of DNA damage by the Comet Assay. They concluded that the use of green tea in the diet could have many benefits for the organism, mainly for its bioactive compounds. However, they suggest that studies are necessary to evaluate its toxicity to better evaluate the safety in its use.

In the article "Effects of type 2 diabetes mellitus induction and of moderate physical exercise on the hepatic metabolism and oxidative stress in Wistar rats" the authors tested the hypothesis that the induction of Type 2 Diabetes Mellitus (T2DM) by the association of streptozotocin and high-fat diet causes hepatic alterations and that physical exercise can influence these alterations. They tested the hypothesis using males Wistar rats and induced T2DM by offering a high-fat group (HF) for 8 weeks and on the $14^{\text {th }}$ received a single dose of streptozotocin $(25 \mathrm{mg} / \mathrm{kg})$. After T2DM induction, a group performed aerobic exercise. They found that the used protocol induced T2DM but not liver diseases or an increase in blood oxidative stress. Besides, physical exercise acted in synergy with high-fat diet and STZ inducing accumulation of triacylglycerol and VLDL in hepatic tissue, an effect that couId either represents lipotoxicity or a protective mechanism to conserve energy.
In the study "L-glutamine supplementation associated with moderate aerobic training improves biometric, glycemic profile and antioxidant defense" the authors aimed to verify whether the L-glutamine supplementation (in its free form) could provide additional improvement in biometric, glycemic and redox parameters in animals undergoing moderate aerobic training (MAT). They found that L-glutamine supplementation increased the gastrocnemius mass and improved the glucose tolerance in animals submitted to MAT. It improved the antioxidant status in gastrocnemius, liver and pancreas, and declined it in adipose tissue in animals undergoing MAT. The drop of adipose antioxidant defense was associated with adiposity, while pancreas antioxidant activity was inversely associated with glucose intolerance.

The editors anticipate this special issue to be of interest to the scientific community. We hope researchers benefit in this issue to make further progress in the understanding of the role of ROS in the development of diseases or for the use of potential therapeutical approaches. The editors would like to thank the authors for submitting their research for publication and the reviewers for their important input and critiques to improve the manuscripts.

Julia Matzenbacher dos Santos

\section{REFERENCES}

DOS SANTOS, Julia M.; TEWARI, Shikha; MENDES, Roberta $\mathrm{H}$. The role of oxidative stress in the development of diabetes mellitus and its complications. Journal of Diabetes Research, v. 2.019, 2019. DOI: 10.1155/2019/4189813

DOS SANTOS, Julia Matzenbacher et al. The role of mitochondrial DNA damage at skeletal muscle oxidative stress on the development of type 2 diabetes. Molecular and $\mathrm{Ce}$ Ilular Biochemistry, v. 449, n. 1-2, p. 251-255, 2018. DOI: 10.1007/s11010-018-3361-5.

JARRETT, S. G. et al. Mitochondrial DNA damage and its potential role in retinal degeneration. Progress in Retinal and Eye Research, v. 27, n. 6, p. 596-607, 2008. DOI: 10.1016/j. preteyeres.2008.09.001.

$\mathrm{JI}$, L. L. et al. Antioxidant enzyme systems in rat liver and skeletal muscle. Influences of selenium deficiency, chronic training, and acute exercise. Archives of Biochemistry and Biophysics, v. 263 , n. 1, p. 150-160, 1988. DOI: 10.1016/0003-9861(88)90623-6.

SANTOS J. M. et al. Diabetic retinopathy, superoxide damage and antioxidants. Curr Pharm Biotechnol., 12(3), p. 35261, 1ㅇ. Mar. 2011. DOI: 10.2174/138920111794480507. PMID: 20939803; PMCID: PMC3214730. 